\title{
Betavoltaics using scandium tritide and contact potential difference
}

\author{
Baojun Liu, ${ }^{1}$ Kevin P. Chen, ${ }^{1, a)}$ Nazir P. Kherani, ${ }^{2}$ Stefan Zukotynski, ${ }^{2}$ and \\ Armando B. Antoniazzi ${ }^{3}$ \\ ${ }^{1}$ Department of Electrical and Computer Engineering, University of Pittsburgh, Pittsburgh, \\ Pennsylvania 15261, USA \\ ${ }^{2}$ Department of Electrical and Computer Engineering, University of Toronto, Toronto, Ontario, \\ Canada M5S $3 G 4$ \\ ${ }^{3}$ Kinectrics Inc., 800 Kipling Ave., Toronto, Ontario, Canada M8Z 6C4
}

(Received 30 October 2007; accepted 4 February 2008; published online 29 February 2008)

\begin{abstract}
Tritium-powered betavoltaic micropower sources using contact potential difference (CPD) are demonstrated. Thermally stable scandium tritide thin films with a surface activity of $15 \mathrm{mCi} / \mathrm{cm}^{2}$ were used as the beta particle source. The electrical field created by the work function difference between the ScT film and a platinum or copper electrode was used to separate the beta-generated electrical charge carriers. Open circuit voltages of 0.5 and $0.16 \mathrm{~V}$ and short circuit current densities of 2.7 and $5.3 \mathrm{nA} / \mathrm{cm}^{2}$ were achieved for gaseous and solid dielectric media-based CPD cells, respectively. (C) 2008 American Institute of Physics. [DOI: 10.1063/1.2887879]
\end{abstract}

Recently, miniaturized radioisotope micropower sources have drawn significant attention because of their potential of providing a long-term energy solution for niche low-power consuming microsystems for a range of applications including exploration and surveillance. ${ }^{1-6}$ A number of nuclear-toelectrical energy conversion integrations, which had been investigated previously, are receiving renewed attention and novel materials and modern microfabrication techniques are being introduced. ${ }^{1,3}$ In recent years, a series of studies have been reported on betavoltaics employing semiconductor $p n$ junction using materials such as amorphous silicon, ${ }^{2}$ porous silicon, ${ }^{4}$ silicon carbide, ${ }^{5,6}$ and gallium arsenide/nitride. ${ }^{1}$ The efficiency and longevity of these battery structures rely on high-quality $p n$ junctions, which are often susceptible to radiation damage and lack structural self-healing resiliency.

One simple method of converting the kinetic energy of ionizing radioemissions into electrical energy is to utilize a contact potential difference (CPD) device produced from materials with dissimilar work functions. An appropriate dielectric medium, in the form of a thin film, gas, or liquid, is sandwiched between the two dissimilar electrodes. An ionizing radiation source, dispersed within the dielectric medium or included in an electrode, provides the energy source for electron-ion pair generation. Charge carriers are separated by the built-in electric field, created by the contact potential difference of the electrodes, generating current in an external load. A CPD-based micropower source is relatively simple to implement and can be integrated for on-chip applications.

Tritium, a radioisotope of hydrogen, is a good candidate for betavoltaic applications given its benign radiation characteristics, its relatively long half-life of 12.3 years, and availability. ${ }^{2,4,7,8}$ Tritium is a pure beta emitter producing energetic electrons with an average energy of $5.7 \mathrm{keV}$ and a maximum energy of $18.6 \mathrm{keV}$. Considering that the threshold electron energy for disruption of the silicon lattice due to knock-on collisions is $20 \mathrm{keV},{ }^{9}$ tritium decay beta particles pose little radiation damage concern for on-chip energy conversion devices. These factors make tritium an appealing power source for on-chip applications. Tritium in the gaseous

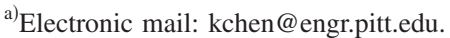

form has been used in self-power lighting and in betavoltaic applications. ${ }^{1,4}$ However, the gaseous form of tritium has a low-power density of $87 \mu \mathrm{W} / \mathrm{cm}^{3}$, not to mention the challenge of hermetic containment of tritium gas and associated hazard of tritium leakage. In this letter, we demonstrate a betavoltaic micropower source using high-density tritium metal hydride foils. Tritium is integrated in one of the electrodes in the form of a stable scandium tritide $\left(\mathrm{ScT}_{x}\right.$, where $x$ is $\mathrm{T}$ to $\mathrm{Sc}$ atom ratio) thin film.

Fabrication of the $\mathrm{ScT}_{x}$ film commenced with the thermal evaporation of scandium; the Sc film, of $300 \mathrm{~nm}$ in thickness, is deposited on a silicon substrate. The Sc film was subsequently tritiated by exposing it to tritium gas for $10 \mathrm{~h}$ at a temperature of $250{ }^{\circ} \mathrm{C}$ and tritium pressure of 10 bars. The high-pressure tritium exposure apparatus used in this experiment is described elsewhere. ${ }^{8}$

To study the stability of the tritiatied $\mathrm{ScT}_{x}$ film, a thermal effusion measurement ${ }^{10,11}$ was performed. Effusion data showed tritium outgassing from the samples beginning at approximately $250{ }^{\circ} \mathrm{C}$, the tritium loading temperature, while the majority of tritium evolution occurred near $600{ }^{\circ} \mathrm{C}$. Thermal effusion experiments provided the concentration of tritium in the $\mathrm{ScT}_{x}$ films of approximately $60 \mathrm{mCi} / \mathrm{cm}^{2}$, which is equivalent to a tritium to scandium atomic ratio $x=1$.

A $3.3 \mathrm{~cm}^{2} \mathrm{ScT}$ on $\mathrm{Si}$ sample was placed in the tritium effusion monitor, in ambient atmosphere at room temperature for a week to monitor the long-term stability of tritium in the film. An average outgassing rate of less than $2 \mathrm{pCi} \mathrm{cm}^{-2} \mathrm{~s}^{-1}$ or $4.2 \times 10^{7}$ atoms $\mathrm{cm}^{-2} \mathrm{~s}^{-1}$ was obtained. Assuming that the outgassing rate is undiminished with time, this is equivalent to a tritium diffusion half-life of greater than 100 years, over eight times the tritium decay half-life. The loss of tritium from the sample due to room temperature outgassing may be thus considered negligible.

Tritium immobilized in ScT film emits beta particles and the betas ionize the gas near the film. A beta particle, traversing in air, with an initial average kinetic energy of $5.7 \mathrm{keV}$ produces approximately 170 electron-ion pairs. A surface activity monitor (SAM) was used to characterize the effective surface activity available for ionization. ${ }^{12}$ A schematic of the 


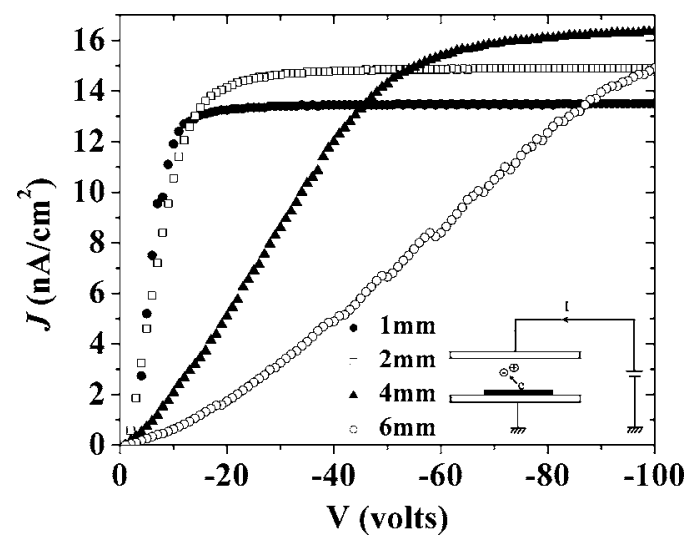

FIG. 1. Ionization current density $(J)$ due to a ScT film sample in air as a function of bias voltage $(V)$. The air gaps are 1,2, 4, and $6 \mathrm{~mm}$, respectively.

SAM monitor is shown in the inset of Fig. 1; the ScT sample, of $2 \mathrm{~cm}^{2}$ area, is placed on the lower electrode. The ionization current density $(J)$ as a function of the applied bias voltage at electrode gaps of $1,2,4$, and $6 \mathrm{~mm}$ is shown in Fig. 1. The saturated current density is approximately $15 \mathrm{nA} / \mathrm{cm}^{2}$. Using the average ionization energy in air of $33.75 \mathrm{eV}$ per ion pair, the effective surface activity of the ScT film is estimated to be $15 \mathrm{mCi} / \mathrm{cm}^{2}$, which is equivalent to an average energy $(5.7 \mathrm{keV})$ beta flux density of $90 \mathrm{pA} / \mathrm{cm}^{2}$ or a power density of $0.5 \mu \mathrm{W} / \mathrm{cm}^{2}$.

To extract the energy of the beta particles, the contact potential difference of two dissimilar metal electrodes was employed to produce an electric field. The ScT film was used as the cathode, as well as the ionization source, while copper was used as the anode. The work functions of pure Sc and $\mathrm{Cu}$ are 3.5 and $4.7 \mathrm{eV}$, respectively. The active plate area was $2.5 \times 1.3 \mathrm{~cm}^{2}$ and the air gaps were varied from 0.05 to $3 \mathrm{~mm}$. A Keithley 617 electrometer was used to measure the voltage and current. The measurement was carried out at room temperature and standard pressure. The $I V$ characteristics of the CPD cell at electrode distances of $0.1,0.3$, and $0.6 \mathrm{~mm}$ are shown in Fig. 2. An open circuit voltage $\left(V_{\mathrm{oc}}\right)$ of $0.5 \mathrm{~V}$ and a short circuit current $\left(I_{\mathrm{sc}}\right)$ of $8.73 \mathrm{nA}$, which is equivalent to a short circuit current density of $2.67 \mathrm{nA} / \mathrm{cm}^{2}$, were measured at a distance of $0.1 \mathrm{~mm}$. This current density is about $20 \%$ of the saturation current density at an air gap of $1 \mathrm{~mm}$ shown in Fig. 1, in recognition of the fact that the saturation current density drops significantly

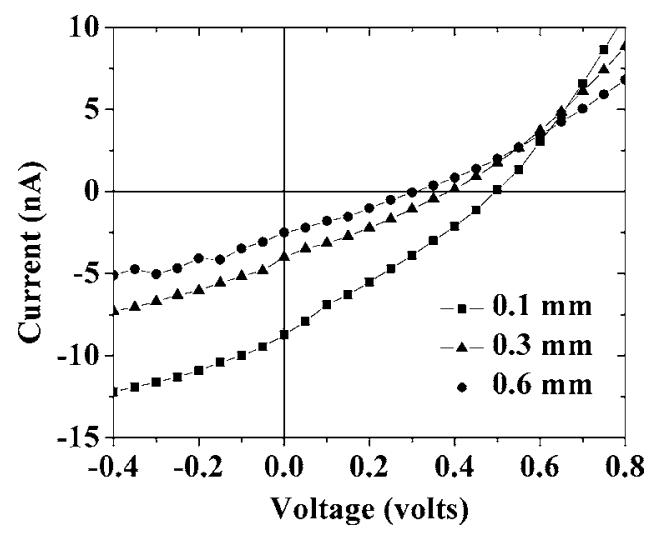

FIG. 2. The $I V$ characteristics of a $2.5 \times 1.3 \mathrm{~cm}^{2} \mathrm{CPD}$ cell in air with electrode separations of $0.1,0.3$, and $0.6 \mathrm{~mm}$, respectively.

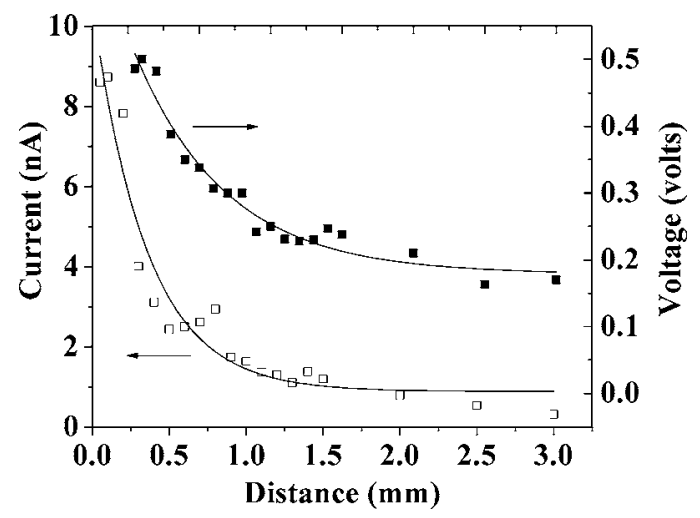

FIG. 3. The short circuit current $\left(I_{\mathrm{sc}}\right)$ and the open circuit voltage $\left(V_{\mathrm{oc}}\right)$ as a function of the electrode separation $(d)$ in a CPD cell in air.

with the decrease of the air gap below $6 \mathrm{~mm}$, which is the maximum travel distance of the tritium beta particles. At an air gap of $0.1 \mathrm{~mm}$, the saturation current density dropped to about half of the value, thus, the CPD cell collected about $40 \%$ of current here. The maximum power point yields $1.3 \mathrm{nW}\left(0.4 \mathrm{nW} / \mathrm{cm}^{2}\right)$, which corresponds to a fill factor of 0.3 . The current may be increased by increasing the gas pressure or/and by using a gas with a lower ionization energy, such as argon.

Both the open circuit voltage $V_{\text {oc }}$ and the short circuit current $I_{\text {sc }}$ decrease with electrode separation $d$ (see Fig. 3). The short circuit current shows a more pronounced drop with distance than the open circuit voltage. This is reasonable considering that the maximum range of tritium beta particles in air is about $6 \mathrm{~mm}$ and that their kinetic energy decays exponentially with the distance traversed. Moreover, electron-ion recombination increases as the electrode separation increases.

In an attempt to further enhance the energy conversion, solid state CPD cells were fabricated and tested.

Intrinsic hydrogenated amorphous silicon $(a-\mathrm{Si}: \mathrm{H})$ was used as the dielectric medium, sandwiched between a platinum $(\mathrm{Pt})$ anode and ScT cathode. Titanium and platinum were sequentially evaporated on a silicon substrate using an e-beam evaporator. The $\mathrm{Ti}$ and $\mathrm{Pt}$ films were approximately 20 and $200 \mathrm{~nm}$ thick, respectively. Subsequently, $a-\mathrm{Si}: \mathrm{H}$ film of $400 \mathrm{~nm}$ thickness was deposited on the Pt film using the dc saddle field plasma-enhanced chemical-vapor deposition technique. ${ }^{2,13}$ The deposition was carried out by decomposing pure silane gas in a glow discharge. The flow rate of silane was 30 SCCM (SCCM denotes cubic centimeter per minute at STP). The deposition chamber pressure was 160 mTorr. The anode potential was $600 \mathrm{~V}$. The anode current was $18 \mathrm{~mA}$. The ion current at the substrate was $0.6 \mathrm{~mA}$. The substrate temperature was $250{ }^{\circ} \mathrm{C}$. The deposition time was $1 \mathrm{~h}$ and $20 \mathrm{~min}$. An array of scandium dots was thermally evaporated onto the $a-\mathrm{Si}: \mathrm{H}$. The dots were of $3 \mathrm{~mm}$ diameter and $300 \mathrm{~nm}$ thickness, each.

A schematic of the CPD cell is illustrated in the inset of Fig. 4. Prior to the tritiation step, each cell was tested for its IV characteristics under darkness and room light illumination. The cells showed good rectification and photovoltaic characteristics. The tested CPD cells were then exposed in tritium gas for $10 \mathrm{~h}$ at a temperature of $250{ }^{\circ} \mathrm{C}$ and a pressure of 10 bars. The resulting scandium tritide film showed a surface activity of $15 \mathrm{mCi} / \mathrm{cm}^{2}$, as discussed earlier. In 


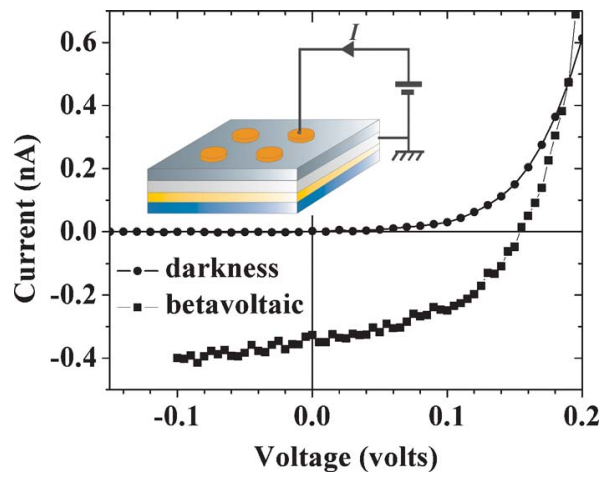

FIG. 4. (Color online) The $I V$ characteristics of a solid CPD cell in darkness before tritiation and betavoltaic performance after tritiation. The solid dielectric is $a-\mathrm{Si}: \mathrm{H}$.

$a-\mathrm{Si}: \mathrm{H}$, the mean ionization energy is reported to be approximately $4.5 \mathrm{eV}$ per electron-hole pair. ${ }^{10}$ The theoretical upper limit for the short circuit current density can be estimated using the following relation: ${ }^{10}$

$$
J_{\mathrm{sc}}=\lambda n_{T} E_{\beta} / \varepsilon,
$$

where $\lambda$ is the decay constant of tritium $\left(1.78 \times 10^{-9} \mathrm{~s}\right)$, $n_{T}$ is the surface activity of the scandium tritide film $\left(15 \mathrm{mCi} / \mathrm{cm}^{2}\right.$, which is equivalent to $3.1 \times 10^{17}$ atoms $/ \mathrm{cm}^{2}$ ), $E_{\beta}$ is the average kinetic energy of the tritium betas $(5.7 \mathrm{keV})$, and $\varepsilon$ represents the ionization energy $(4.5 \mathrm{eV})$. Equation (1) yields a $J_{\mathrm{sc}}$ of $110 \mathrm{nA} / \mathrm{cm}^{2}$.

After the tritiation, dark $I V$ measurements were carried out. A typical result is shown in Fig. 4. Powered by the ScT film, an open circuit voltage of $160 \mathrm{mV}$ and a short circuit current of $370 \mathrm{pA}$ were measured. The area of the ScT disk is about $7 \mathrm{~mm}^{2}$. The maximum power point was measured to be $18 \mathrm{pW}\left(0.26 \mathrm{nW} \mathrm{cm}^{2}\right)$. The short circuit current density was measured to be $5.3 \mathrm{nA} / \mathrm{cm}^{2}$, which was $4.8 \%$ of the theoretical limit calculated by Eq. (1). Owing to a smaller ionization energy in $a-\mathrm{Si}: \mathrm{H}$, the short circuit current density was doubled compared to that of the cell with the gaseous dielectric, though the overall output power density was not improved owing to the lower open circuit voltage.

Both CPD cells with the gaseous and semiconductor dielectrics produced electrical power at the nanowatt level. The energy conversion efficiency is found to be about $0.1 \%$. This is comparable to other reported betavoltaic devices powered by tritium ${ }^{2,4}$ and less than that of the recently reported $\mathrm{SiC}$ pn-junction cell using high energy betas from ${ }^{63} \mathrm{Ni}$ and ${ }^{33} \mathrm{P} ., 6$ In the gaseous CPD cells, the low-power density and conversion efficiency is attributed to the relatively high ionization energy, while in the $a$-Si:H CPD cells, the lower performance is attributed to the low built-in field and associated higher recombination rate.

In summary, tritium based nuclear batteries using $\mathrm{ScT}_{x}$ films have been demonstrated. The batteries utilize air and $a$-Si:H film as dielectric media, displaying short circuit current densities of $40 \%$ and $4.8 \%$ of the theoretical limits, respectively. The batteries provide power with a projected lifetime comparable to the half-life of tritium and can be operated at temperatures approaching $250{ }^{\circ} \mathrm{C}$. The appropriateness of $\mathrm{ScT}_{x}$ is also evident from its low tritium vapor pressure at elevated temperatures. ${ }^{14}$ The output power density can be further enhanced by increasing the surface activity of the $\mathrm{ScT}_{x}$ film using a textured surface as well as through improved passivation of the CPD cell. It is suggested that using a stacked series-connected cell geometry, a tritium battery based on the CPD principle could be realized with a continuous output power of $1 \mu \mathrm{W}$ at a cell volume of $\sim 1 \mathrm{~cm}^{3}$.

This work was supported by NSF Grant No. 0556086 and NSERC discovery grants. The authors wish to thank Dr. Davit Yeghikyan for his help in the deposition of the $a-\mathrm{Si}: \mathrm{H}$ film and Mr. Clive Morton for his assistance in tritium loading.

${ }^{1}$ K. E. Bower, Y. A. Barbanel, Y. G. Shreter, and G. W. Bohnert, Polymer, Phosphors, and Voltaics for Radioisotope Microbatteries (CRC, Boca Raton, FL, 2002).

${ }^{2}$ T. Kosteski, N. P. Kherani, P. Stradins, F. Gaspari, W. T. Shmayda, L. S. Sidhu, and S. Zukotynski, IEE Proc.: Circuits Devices Syst. 150, 274 (2003).

${ }^{3}$ A. Lal and J. Blanchar, IEEE Spectrum 41, 36 (2004).

${ }^{4}$ W. Sun, N. P. Kherani, K. D. Hirschman, L. L. Gadeken, and P. M. Fauchet, Adv. Mater. (Weinheim, Ger.) 17, 1230 (2005).

${ }^{5}$ M. V. S. Chandrashekhar, C. I. Thomas, H. Li, M. G. Spencer, and A. Lal, Appl. Phys. Lett. 88, 033506 (2006).

${ }^{6}$ C. J. Eiting, V. Krishnamoorthy, S. Rodgers, T. George, J. D. Robertson, and J. Brockman, Appl. Phys. Lett. 88, 064101 (2006).

${ }^{7}$ B. Liu, K. P. Chen, N. P. Kherani, T. Kosteski, S. Costea, S. Zukotynski, and A. B. Antoniazzi, Appl. Phys. Lett. 89, 044104 (2006).

${ }^{8}$ B. Liu, K. P. Chen, N. P. Kherani, S. Zukotynski, and A. B. Antoniazzi, Appl. Phys. Lett. 88, 134101 (2006).

${ }^{9}$ J. R. Srour, C. J. Marshall, and P. W. Marshall, IEEE Trans. Nucl. Sci. 50, 653 (2003).

${ }^{10}$ T. Kosteski, Ph.D thesis, University of Toronto, 2001.

${ }^{11}$ N. P. Kherani, B. Liu, K. Virk, T. Kosteski, F. Gaspari, W. T. Shmayda, S. Zukotynski, and K. P. Chen, J. Appl. Phys. 103, 024906 (2008).

${ }^{12}$ N. P. Kherani and W. T. Shmayda, Fusion Technol. 28, 893 (1995).

${ }^{13}$ N. P. Kherani, T. Kosteski, S. Zukotynski, and W. T. Shmayda, Fusion Technol. 28, 1609 (1995).

${ }^{14}$ A. W. P. Poon, R. J. Komar, C. E. Waltham, M. C. Browne, R. G. H. Robertson, N. P. Kherani, and H. B. Mak, Nucl. Instrum. Methods Phys. Res. A 452, 115 (2000). 
Applied Physics Letters is copyrighted by the American Institute of Physics (AIP). Redistribution of journal material is subject to the AIP online journal license and/or AIP copyright. For more information, see http://ojps.aip.org/aplo/aplcr.jsp 